\title{
On the Uniqueness of a Certain Thin Near Octagon (or Partial 2-Geometry, or Parallelism) Derived from the Binary Golay Code
}

\author{
A. E. BROUWER
}

DEDICATED TO JESSIE MACWILLIAMS ON THE OCCASION OF HER RETIREMENT FROM BELL LABORATORIES

\begin{abstract}
The question of the uniqueness of a certain combinatorial structure has arisen in three contexts: a) is the regular near octagon with parameters $\left(s, t_{2}, t_{3}, t\right)=(1,1,2,23)$ unique [5]? b) is the partial 2-geometry with nexus three and blocksize 24 unique [2]? c) is there a unique graph such that it is the graph of a parallelism of $\left(\begin{array}{c}24 \\ 4\end{array}\right)$ with respect to any vertex [1]? We observe that these questions are equivalent and give an affirmative answer. In fact, we prove a more general theorem, showing the truth of a conjecture by Cameron.
\end{abstract}

\section{INTRODUCTION}

$\mathrm{F}$ OR the definition of near $n$-gon (see [5], [6]). We shall only use thin regular near $2 d$-gons which are nothing but distance regular bipartite graphs of diameter $d$. If $d(x, y)=i$ then let $c_{i}:=\mid\{z \mid z \sim y$ and $d(z, x)=i-1\} \mid$ (where $\sim$ denotes adjacency). By distance regularity $c_{i}$ does not depend on the choice of $x$ and $y$. Write $k:=c_{d}$, the valency of the graph.

A partial $\lambda$-geometry with nexus $e$ is an incidence structure with $b$ blocks and $v$ points which satisfies:

a) two distinct points are on 0 or $\lambda$ common blocks;

b) two distinct blocks have 0 or $\lambda$ common points;

c) given a point $x$ not on a block $B$, there are precisely $e$ blocks on $x$ meeting $B$;

d) no point is on all blocks and no block contains all points;

e) all blocks have size $k$ and all points are in $r$ blocks, where $k, r>\lambda$, (see [1], [2]).

If $\lambda>1$ then one may weaken e) to each block has more than $\lambda$ points and each point is on more than $\lambda$ blocks and obtain the existence of $k$ and $r$ and moreover that $k=r$. Assume $\lambda>1$ and $e<k$ (when $e=k$ we have a symmetric BIBD).

One immediately verifies that the bipartite graph with points and blocks as vertices and incidence as adjacency is

Manuscript received April 12, 1982; revised June 8, 1982.

The author is with the Mathematical Center, Kruislaan 413, Amsterdam, The Netherlands. distance regular with $\left(c_{1}, c_{2}, c_{3}, c_{4}\right)=(1, \lambda, e, k)$, i.e., we have a thin regular near octagon. The converse also being clear we see that the concepts of thin regular near 8-gon (with $c_{2}>1$ ) and partial $\lambda$-geometry with nexus $e$ (with $\lambda>1$ and $e<k$ ) are equivalent.

\section{UNIQUENESS}

Let $\Gamma$ be a distance regular bipartite graph with diameter $d \geqslant 4$ and $c_{i}=i(1 \leqslant i \leqslant d-1)$.

Lemma: Let $2^{j}$ be a $j$-cube (vertices: binary vectors of length $j$, edges: pairs of vectors differing by a unit vector) and $\pi$ be a map sending 0 to $x_{0} \in \Gamma$ and the unit vectors to (distinct) neighbors of $x_{0}$ in $\Gamma$. Then $\pi$ can be extended in a unique way to a map $\pi: 2^{j} \rightarrow \Gamma$ preserving adjacency and squares. Moreover, $\pi$ is injective when restricted to $(2 d-$ 1)-cubes.

Proof (cf. [1, th. 5.11 (i) ]): First observe that two points at distance three in $\Gamma$ determine a 3-cube: each has three neighbors at distance two from the other, and on these six points we have a regular bipartite graph of valency two, hence a hexagon, completing the cube. It follows easily that a 2-claw determines a square and a 3-claw a 3-cube.

We define $\pi(v)$ by induction on the number of nonzero coordinates (the weight) of $v$. If the weight is zero or one $\pi(v)$ is prescribed. If the weight is two so that $v=e+e^{\prime}$ for two unit vectors $e, e^{\prime}$, then let $\pi(v)$ be the common neighbor of $\pi(e)$ and $\pi\left(e^{\prime}\right)$ distinct from $\pi(\mathbf{0})$.

Suppose $\pi(\boldsymbol{v})$ defined for vectors $v$ of weight less than $i$, and let $v$ have weight $i \geqslant 3: v=\sum_{h=1}^{i} e_{h}$ for distinct unit vectors $\boldsymbol{e}_{h}$. Then we can define $\pi(\boldsymbol{v})$ as the common neighbor of $\pi\left(v-e_{1}\right)$ and $\pi\left(v-e_{2}\right)$ distinct from $\pi(v-$ $\left.e_{1}-e_{2}\right)$. For $h>2$ the points $\pi(v)$ and $\pi\left(v-e_{1}-e_{2}-\right.$ $\boldsymbol{e}_{h}$ ) have distance three, hence determine a 3-cube, and we find that $\pi(v)$ is adjacent to $\pi\left(v-e_{h}\right)$ as was required. Thus, using only $c_{2}=2$ and $c_{3}=3$ we find a unique map $\pi$ satisfying all requirements and injective on 3-cubes. If we 
also have $c_{i}=i(2 \leqslant i \leqslant h)$ then one easily sees that $\pi$ is still injective on $(2 h+1)$-cubes: if two vectors have the same image then by choosing the origin appropriately one may suppose that they have disjoint supports of equal weights at most $h$ (note that $\Gamma$ is bipartite and $2^{j}$ is connected so that vectors with the same image have even distance), but inside a radius of $h$ nothing can happen.

Now let $k$ be the valency of $\Gamma$ and apply the Lemma to find a labeling of $\Gamma$ with vectors from $2^{k}$. The number of vertices of $\Gamma$ is

$$
|V(\Gamma)|=1+k+\left(\begin{array}{l}
k \\
2
\end{array}\right)+\cdots+\left(\begin{array}{c}
k \\
d-1
\end{array}\right)+\frac{d}{k}\left(\begin{array}{l}
k \\
d
\end{array}\right),
$$

so that on the average each point gets $2^{k} / V(\Gamma) \mid$ labels. But the collection of labels of a point $x$ is a binary code $e_{x}$ with word length $k$ and minimum distance at least $2 d$, and this average is just the trivial upper bound for the cardinality of such a code when one observes that spheres of radius $d-1$ around codewords are disjoint, and a vector can have distance $d$ to at most $k / d$ distinct codewords. Consequently each point has exactly $2^{k} /|V(\Gamma)|$ labels, and each $\bigodot_{x}$ is an extended perfect code. By the classification of perfect codes (see e.g., [4, ch. 6, th. 33]) it follows that we have one of the following three possibilities:

a) $\left|e_{x}\right|=1$, the code is trivial. Now $d=k,|V(\Gamma)|=2^{k}$ and we have a $k$-cube.

b) $\left|e_{x}\right|=2$, a repetition code. Now $d=\frac{1}{2} k,|V(\Gamma)|=$ $2^{k-1}$ and we have a half $k$-cube (i.e., a $k$-cube with antipodal vertices identified).

c) $\left|e_{x}\right|=2^{12}$, a code isomorphic to the extended binary Golay code $e$. Now $k=24, d=4$ and all codes $e_{x}$ are translates of each other (for: suppose $x \sim y$. For each $v \in \bigodot_{x}$ there is a unique $v^{\prime} \in \bigodot_{y}$ with $d\left(v, v^{\prime}\right)=$
1. The map $v \rightarrow v^{\prime}$ changes distances by at most two, but since all distances are multiples of four it must preserve distances. Considering triples of vectors of weight $8 u, v, w$ in $e_{x}$ such that $u+v+w$ is the all-one vector one sees that this map is a translation over a vector of weight one). Our graph $\Gamma$ is the graph

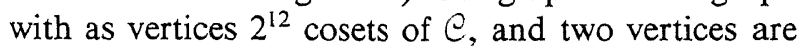
adjacent if and only if the corresponding cosets contain vectors differing by a unit vector.

This settles question 5.7 in [1, p. 94]-there also $d=3$ is allowed, but in this case it is given that 3-claws generate 3 -cubes and our reasoning still works.

Let us formulate a theorem.

Theorem: Let $\Gamma$ be a distance regular bipartite graph with diameter $d \geqslant 3$, valency $k$ and parameters $c_{i}=i(1 \leqslant$ $i \leqslant d-1$ ). If $d=3$ then suppose additionally that any 3-claw generates a 3-cube (in the sense of [1, p. 91]). Then $\Gamma$ is a $d$-cube, a half $2 d$-cube, or we have $d=4, k=24$, and $\Gamma$ is the graph of the sextet parallelism (or, in other words, is the thin near octagon derived from the extended binary Golay code as described above).

\section{REFERENCES}

[1] P. J. Cameron, Parallelisms of Complete Designs. Cambridge, England: Cambridge University Press 1976.

[2] P. J. Cameron and D. A. Drake, "Partial $\lambda$-geometries of small nexus," Ann. Discrete Math., vol. 6, pp. 19-29, 1980.

[3] D. A. Drake, "Partial $\lambda$-geometries and generalized Hadamard matrices over groups," to appear.

[4] F. J. MacWilliams and N. J. A. Sloane, The Theory of Error-Correcting Codes. Amsterdam: North Holland, 1977.

[5] S. Shad and E. Shult, "The near n-gon geometries," to appear.

[6] E. Shult and A. Yanushka, "Near n-gons and line systems," Geometriae Dedicata, vol. 9, pp. 1-72, 1980. 